Supplement of Atmos. Chem. Phys., 19, 8269-8296, 2019

https://doi.org/10.5194/acp-19-8269-2019-supplement

(C) Author(s) 2019. This work is distributed under

the Creative Commons Attribution 4.0 License.

(c) (1)

Supplement of

\title{
IAP-AACM v1.0: a global to regional evaluation of the atmospheric chemistry model in CAS-ESM
}

\section{Ying Wei et al.}

Correspondence to: Zifa Wang (zifawang@mail.iap.ac.cn)

The copyright of individual parts of the supplement might differ from the CC BY 4.0 License. 
Table S1. The list of observation sites information

\begin{tabular}{|c|c|c|c|}
\hline Number & Site name (ID) & Longitude & Latitude \\
\hline \multicolumn{4}{|c|}{ WDCGG Dataset } \\
\hline 1 & asc 107 s00.noa & -14.42 & -7.92 \\
\hline 2 & ask123n00.noa & 5.63 & 23.27 \\
\hline 3 & azr638n00.noa & -27.37 & 38.77 \\
\hline 4 & bhd541s00.noa & 174.87 & -41.4 \\
\hline 5 & bkt500s00.bmg & 100.32 & -0.2 \\
\hline 6 & bmw432n00.noa & -64.87 & 32.27 \\
\hline 7 & brw471n00.noa & -156.6 & 71.32 \\
\hline 8 & cba455n00.noa & -162.72 & 55.2 \\
\hline 9 & cfa519s00.csi & 147.05 & -19.3 \\
\hline 10 & cgo540s00.csi & 144.68 & -40.7 \\
\hline 11 & cmn644n00.isa & 10.7 & 44.18 \\
\hline 12 & cpt134s00.saw & 18.48 & -34.4 \\
\hline 13 & crz146s00.noa & 51.85 & -46.5 \\
\hline 14 & cvo116n00.uyr & -24.87 & 16.85 \\
\hline 15 & cya766s00.csi & 110.53 & -66.3 \\
\hline 16 & egb444n01.ec. & -79.78 & 44.23 \\
\hline 17 & eic327s00.noa & -109.45 & -27.1 \\
\hline 18 & et1454n00.ec. & -104.98 & 54.35 \\
\hline 19 & glh636n00.uml & 14.22 & 36.07 \\
\hline 20 & gmi513n00.noa & 144.78 & 13.43 \\
\hline 21 & hba775s00.noa & -26.5 & -75.6 \\
\hline 22 & hpb647n00.noa & 11.02 & 47.8 \\
\hline 23 & hun646n00.noa & 16.65 & 46.95 \\
\hline 24 & ice663n00.noa & -20.28 & 63.4 \\
\hline 25 & izo128n00.aem & -16.5 & 28.3 \\
\hline 26 & key425n00.noa & -80.2 & 25.67 \\
\hline 27 & kos649n00.chm & 15.08 & 49.58 \\
\hline 28 & kum519n00.noa & -154.82 & 19.52 \\
\hline 29 & kvv646n00.ars & 14.53 & 46.3 \\
\hline 30 & lef445n00.noa & -90.27 & 45.92 \\
\hline 31 & $1 \ln 223$ n00.noa & 120.87 & 23.47 \\
\hline 32 & lmp635n00.noa & 12.63 & 35.52 \\
\hline 33 & maa767s00.csi & 62.87 & -67.6 \\
\hline 34 & mex419n00.noa & -97.17 & 19.98 \\
\hline 35 & mhd653n00.noa & -9.9 & 53.33 \\
\hline 36 & mid528n00.noa & -177.37 & 28.2 \\
\hline 37 & mlo519n00.csi & -155.58 & 19.54 \\
\hline 38 & $\mathrm{mnm} 224 \mathrm{n} 00 . \mathrm{jma}$ & 153.98 & 24.28 \\
\hline 39 & mqa554s00.csi & 158.97 & -54.5 \\
\hline 40 & nat306s00.noa & -35.2 & -6 \\
\hline
\end{tabular}




\begin{tabular}{|c|c|c|c|}
\hline Number & Site name (ID) & Longitude & Latitude \\
\hline 41 & nmb123s00.noa & 15.02 & -23.6 \\
\hline 42 & nwr440n01.noa & -105.59 & 40.05 \\
\hline 43 & oxk650n00.noa & 11.8 & 50.03 \\
\hline 44 & pal667n00.noa & 24.12 & 67.97 \\
\hline 45 & poc900n00.noa & -155 & 0 \\
\hline 46 & poc $905 n 00$. noa & -151 & 5 \\
\hline 47 & poc905s00.noa & -159 & -5 \\
\hline 48 & poc910n00.noa & -149 & 10 \\
\hline 49 & poc910s00.noa & -161 & -10 \\
\hline 50 & poc915n00.noa & -145 & 15 \\
\hline 51 & poc915s00.noa & -171 & -15 \\
\hline 52 & poc920n00.noa & -141 & 20 \\
\hline 53 & poc920s00.noa & -174 & -20 \\
\hline 54 & poc925n00.noa & -139 & 25 \\
\hline 55 & poc925s00.noa & -171 & -25 \\
\hline 56 & poc930n00.noa & -135 & 30 \\
\hline 57 & poc930s00.noa & -176 & -30 \\
\hline 58 & psa764s00.noa & -64 & -64.9 \\
\hline 59 & rig646n00.emp & 8.45 & 46.07 \\
\hline 60 & rpb413n00.noa & -59.43 & 13.17 \\
\hline 61 & ryo239n00.jma & 141.82 & 39.03 \\
\hline 62 & sey104s00.noa & 55.17 & -4.67 \\
\hline 63 & sgp436n00.noa & -97.5 & 36.78 \\
\hline 64 & smo514s00.noa & -170.57 & -14.2 \\
\hline 65 & spo789s00.csi & -24.8 & -90 \\
\hline 66 & sum672n00.noa & -38.48 & 72.58 \\
\hline 67 & syo769s00.noa & 39.58 & -69 \\
\hline 68 & tap236n00.noa & 126.12 & 36.72 \\
\hline 69 & thd441n00.noa & -124.15 & 41.05 \\
\hline 70 & ush354s00.noa & -68.31 & -54.9 \\
\hline 71 & uta439n00.noa & -113.72 & 39.88 \\
\hline 72 & uum244n00.noa & 111.08 & 44.45 \\
\hline 73 & wis631n00.noa & 34.87 & 31.12 \\
\hline 74 & wlg236n00.cma & 100.9 & 36.28 \\
\hline 75 & yon224n00.jma & 123.02 & 24.47 \\
\hline 76 & zep678n00.noa & 11.88 & 78.9 \\
\hline 77 & $\operatorname{dig} 654$ n00.ioe & 22.07 & 54.15 \\
\hline 78 & glh636n00.uml & 14.22 & 36.07 \\
\hline 79 & irb645n00.ars & 14.87 & 45.57 \\
\hline 80 & kos649n00.chm & 15.08 & 49.58 \\
\hline 81 & kps646n00.hms & 19.55 & 46.97 \\
\hline 82 & pay646n00.emp & 6.95 & 46.82 \\
\hline 83 & rcv656n00.1hm & 21.17 & 56.16 \\
\hline
\end{tabular}




\begin{tabular}{|c|c|c|c|}
\hline Number & Site name (ID) & Longitude & Latitude \\
\hline 84 & rig646n00.emp & 8.45 & 46.07 \\
\hline 85 & amy236n00.kma & 126.32 & 36.53 \\
\hline 86 & $\operatorname{arh} 777 \mathrm{~s} 00 . n o a$ & 166.67 & -77.8 \\
\hline 87 & ask123n00.onm & 5.63 & 23.27 \\
\hline 88 & bhd541s00.niw & 174.87 & -41.4 \\
\hline 89 & bmw432n00.noa & -64.87 & 32.27 \\
\hline 90 & brw471n00.noa & -156.6 & 71.32 \\
\hline 91 & cpt134s00.saw & 18.48 & -34.4 \\
\hline 92 & cvo116n00.uyr & -24.87 & 16.85 \\
\hline 93 & glh636n00.uml & 14.22 & 36.07 \\
\hline 94 & irb645n00.ars & 14.87 & 45.57 \\
\hline 95 & jfj646n00.emp & 7.99 & 46.55 \\
\hline 96 & kos649n00.chm & 15.08 & 49.58 \\
\hline 97 & kps646n00.hms & 19.55 & 46.97 \\
\hline 98 & kvk646n00.ars & 15.1 & 46.12 \\
\hline 99 & kvv646n00.ars & 14.53 & 46.3 \\
\hline 100 & lau545s00.noa & 169.67 & -45 \\
\hline 101 & mhd653n00.nui & -9.9 & 53.33 \\
\hline 102 & mlo519n00.noa & -155.58 & 19.54 \\
\hline 103 & mnm224n00.jma & 153.98 & 24.28 \\
\hline 104 & nmy770s00.awi & -8.25 & -70.7 \\
\hline 105 & nwr440n00.noa & -105.54 & 40.04 \\
\hline 106 & pay646n00.emp & 6.95 & 46.82 \\
\hline 107 & prs645n00.rse & 7.7 & 45.93 \\
\hline 108 & rig646n00.emp & 8.45 & 46.07 \\
\hline 109 & rpb413n00.noa & -59.43 & 13.17 \\
\hline 110 & ryo239n00.jma & 141.82 & 39.03 \\
\hline 111 & smo514s00.noa & -170.57 & -14.2 \\
\hline 112 & spo789s00.noa & -24.8 & -90 \\
\hline 113 & sum672n00.noa & -38.48 & 72.58 \\
\hline 114 & syo769s00.jma & 39.58 & -69 \\
\hline 115 & thd441n00.noa & -124.15 & 41.05 \\
\hline 116 & tkb236n10.jma & 140.13 & 36.05 \\
\hline 117 & tll330s00.dmc & -70.8 & -30.2 \\
\hline 118 & ush354s00.smn & -68.31 & -54.9 \\
\hline 119 & vdl664n00.ivl & 19.77 & 64.25 \\
\hline 120 & yon224n00.jma & 123.02 & 24.47 \\
\hline 121 & zrn646n00.ars & 15 & 46.43 \\
\hline 122 & zsn657n00.lhm & 25.54 & 57.08 \\
\hline 123 & $\operatorname{dig} 654 \mathrm{n} 00$. ioe & 22.07 & 54.15 \\
\hline 124 & glh636n00.uml & 14.22 & 36.07 \\
\hline 125 & irb645n00.ars & 14.87 & 45.57 \\
\hline 126 & jfj646n00.emp & 7.99 & 46.55 \\
\hline
\end{tabular}




\begin{tabular}{|c|c|c|c|}
\hline Number & Site name (ID) & Longitude & Latitude \\
\hline 127 & kos649n00.chm & 15.08 & 49.58 \\
\hline 128 & kps646n00.hms & 19.55 & 46.97 \\
\hline 129 & pay646n00.emp & 6.95 & 46.82 \\
\hline 130 & rcv656n00.1hm & 21.17 & 56.16 \\
\hline 131 & rig646n00.emp & 8.45 & 46.07 \\
\hline \multicolumn{4}{|c|}{ EANET } \\
\hline num & staid & lon & lat \\
\hline 1 & PhnomPenh & 104.83 & 11.55 \\
\hline 2 & Jakarta & 106.83 & -6.18 \\
\hline 3 & Serpong & 106.57 & 6.25 \\
\hline 4 & Bandung & 107.58 & 6.9 \\
\hline 5 & Rishiri & 141.2 & 45.12 \\
\hline 6 & Ochishi & 145.5 & 43.15 \\
\hline 7 & Tappi & 140.35 & 41.25 \\
\hline 8 & Sado-seki & 138.4 & 38.23 \\
\hline 9 & Ijira & 136.68 & 35.57 \\
\hline 10 & Oki & 133.18 & 36.28 \\
\hline 11 & Banryu & 131.8 & 34.68 \\
\hline 12 & Hedo & 128.25 & 26.87 \\
\hline 13 & Ogasawara & 142.22 & 27.08 \\
\hline 14 & PetalingJaya & 101.65 & 3.1 \\
\hline 15 & DanumValley & 117.85 & 4.98 \\
\hline 16 & Yangon & 96.12 & 16.5 \\
\hline 17 & Kanghwa & 126.28 & 37.7 \\
\hline 18 & Cheju & 126.17 & 33.3 \\
\hline 19 & Imsil & 127.18 & 35.6 \\
\hline 20 & Listvyanka & 104.9 & 51.85 \\
\hline 21 & Irkutsk & 104.25 & 52.23 \\
\hline 22 & Primorskaya & 132.12 & 43.7 \\
\hline 23 & Bangkok & 100.53 & 13.77 \\
\hline 24 & Khanchanaburi & 98.58 & 14.77 \\
\hline 25 & ChiangMai & 98.93 & 18.77 \\
\hline 26 & NakhonRatchasima & 101.88 & 14.45 \\
\hline 27 & Hanoi & 105.85 & 21.02 \\
\hline 28 & HoaBinh & 105.33 & 20.82 \\
\hline 29 & Tokyo & 139.75 & 35.68 \\
\hline 30 & NakhonRatchasima & 101.88 & 14.45 \\
\hline 31 & Mt.Sto.Tomas 1 & 120.6 & 6.42 \\
\hline 32 & Hongwen & 118.13 & 24.47 \\
\hline 33 & XiangZhou & 113.57 & 22.27 \\
\hline 34 & Kototabang & 100.32 & 0.2 \\
\hline 35 & Ulaanbaatar & 106.82 & 47.9 \\
\hline 36 & Samutprakarn & 100.57 & 13.73 \\
\hline
\end{tabular}




\begin{tabular}{|c|c|c|c|}
\hline Number & Site name (ID) & Longitude & Latitude \\
\hline 37 & Mondy & 101 & 51.67 \\
\hline 38 & Happo & 137.8 & 36.7 \\
\hline 39 & MtStoTomas & 120.6 & 16.42 \\
\hline 40 & Khanchanaburi & 98.58 & 14.77 \\
\hline \multicolumn{4}{|c|}{ EMEP } \\
\hline 1 & Waldhof & 10.76 & 52.8 \\
\hline 2 & Schauinsland & 7.91 & 47.91 \\
\hline 3 & Neuglobsow & 13.03 & 53.17 \\
\hline 4 & Lahemaa & 25.9 & 59.5 \\
\hline 5 & Uto & 21.38 & 59.78 \\
\hline 6 & Virolahti & 27.69 & 60.53 \\
\hline 7 & Pallas & 24.24 & 68 \\
\hline 8 & K-puszta & 19.58 & 46.97 \\
\hline 9 & Oak & -6.92 & 52.87 \\
\hline 10 & Malin & -7.34 & 55.38 \\
\hline 11 & Carnsore & -6.36 & 52.18 \\
\hline 12 & Rucava & 21.17 & 56.16 \\
\hline 13 & Birkenes & 8.25 & 58.39 \\
\hline 14 & Karvatn & 8.88 & 62.78 \\
\hline 15 & Zeppelin & 11.89 & 78.91 \\
\hline 16 & Hurdal & 11.08 & 60.37 \\
\hline 17 & Jarczew & 21.97 & 51.81 \\
\hline 18 & Sniezka & 15.74 & 50.74 \\
\hline 19 & Leba & 17.53 & 54.75 \\
\hline 20 & Diabla & 22.07 & 54.15 \\
\hline 21 & Danki & 37.8 & 54.9 \\
\hline 22 & Iskrba & 14.87 & 45.57 \\
\hline 23 & Starina & 22.27 & 49.05 \\
\hline 24 & Melpitz_l & 12.93 & 51.53 \\
\hline 25 & Ispra & 8.63 & 45.8 \\
\hline 26 & Cabauw & 4.92 & 51.97 \\
\hline 27 & Illmitz & 16.77 & 47.77 \\
\hline 28 & Vorhegg & 12.97 & 46.68 \\
\hline 29 & Zoebelboden & 14.44 & 47.84 \\
\hline 30 & Payerne & 6.94 & 46.81 \\
\hline 31 & Tanikon & 8.9 & 47.48 \\
\hline 32 & Chaumont & 6.98 & 47.05 \\
\hline 33 & Rigi & 8.46 & 47.07 \\
\hline 34 & Churanov & 13.6 & 49.07 \\
\hline 35 & Westerland & 8.31 & 54.93 \\
\hline 36 & Zingst & 12.73 & 54.43 \\
\hline 37 & Harwell & -1.32 & 51.57 \\
\hline 38 & Auchencorth & -3.24 & 55.79 \\
\hline
\end{tabular}




\begin{tabular}{|c|c|c|c|}
\hline Number & Site name (ID) & Longitude & Latitude \\
\hline 39 & Kamenicki & 21.95 & 43.4 \\
\hline 40 & Schmucke & 10.77 & 50.65 \\
\hline 41 & San & -4.35 & 39.55 \\
\hline 42 & Cabo & 3.32 & 42.32 \\
\hline 43 & Zarra & -1.1 & 39.09 \\
\hline 44 & Penausende & -5.87 & 41.28 \\
\hline 45 & Els & 0.72 & 41.4 \\
\hline 46 & Rao & 11.91 & 57.39 \\
\hline \multicolumn{4}{|c|}{ IMPROVE } \\
\hline 1 & ACAD1 & -68.261 & 44.377 \\
\hline 2 & BLMO1 & -96.191 & 43.716 \\
\hline 3 & BRMA1 & -70.729 & 44.107 \\
\hline 4 & CEBL1 & -99.763 & 38.77 \\
\hline 5 & DENA1 & -148.968 & 63.723 \\
\hline 6 & EVER1 & -80.681 & 25.391 \\
\hline 7 & GAAR1 & -151.517 & 66.903 \\
\hline 8 & GRRI1 & -91.405 & 43.937 \\
\hline 9 & HEGL1 & -92.922 & 36.614 \\
\hline 10 & KALM1 & -124.059 & 42.552 \\
\hline 11 & LOST1 & -102.402 & 48.642 \\
\hline 12 & MING1 & -90.143 & 36.972 \\
\hline 13 & NEBR1 & -100.339 & 41.889 \\
\hline 14 & OWVL1 & -118.331 & 37.361 \\
\hline 15 & PMRF1 & -72.869 & 44.528 \\
\hline 16 & RAFA1 & -120.007 & 34.734 \\
\hline 17 & SAGO1 & -116.913 & 34.194 \\
\hline 18 & SENE1 & -85.95 & 46.289 \\
\hline 19 & SIME1 & -160.506 & 55.325 \\
\hline 20 & TALL1 & -96.56 & 38.434 \\
\hline 21 & TRIN1 & -122.805 & 40.786 \\
\hline 22 & WHIT1 & -105.535 & 33.469 \\
\hline 23 & ZICA1 & -113.151 & 37.198 \\
\hline \multicolumn{4}{|c|}{ EPA } \\
\hline 1 & 10730023 & -86.82 & 33.55 \\
\hline 2 & 40128000 & -113.56 & 34.24 \\
\hline 3 & 60530002 & -121.64 & 36.7 \\
\hline 4 & 60831008 & -120.05 & 34.49 \\
\hline 5 & 90090027 & -72.9 & 41.3 \\
\hline 6 & 120573002 & -82.54 & 27.89 \\
\hline 7 & 120860033 & -80.16 & 25.73 \\
\hline 8 & 170310022 & -87.64 & 41.88 \\
\hline 9 & 171190024 & -90.16 & 38.61 \\
\hline 10 & 180570007 & -85.77 & 39.29 \\
\hline
\end{tabular}




\begin{tabular}{|c|c|c|c|}
\hline 11 & 191770006 & -92.01 & 40.7 \\
\hline 12 & 201330003 & -95.48 & 37.68 \\
\hline 13 & 360610079 & -73.9 & 40.82 \\
\hline 14 & 380171004 & -96.86 & 46.93 \\
\hline 15 & 380250003 & -102.53 & 47.31 \\
\hline 16 & 410510080 & -122.6 & 45.5 \\
\hline 17 & 420010001 & -77.31 & 39.92 \\
\hline 18 & 420031008 & -79.73 & 40.61 \\
\hline 19 & 450450015 & -82.41 & 34.84 \\
\hline 20 & 460990006 & -96.7 & 43.55 \\
\hline 21 & 461030020 & -103.27 & 44.09 \\
\hline 22 & 461270001 & -96.71 & 42.75 \\
\hline 23 & 550870009 & -88.81 & 45.56 \\
\hline 24 & 560030003 & -108.39 & 44.84 \\
\hline 25 & 20200018 & -149.82 & 61.21 \\
\hline 26 & 21100004 & -134.57 & 58.39 \\
\hline 27 & 21221006 & -151.69 & 59.46 \\
\hline 28 & 21700008 & -149.03 & 61.53 \\
\hline 29 & 40131003 & -111.87 & 33.41 \\
\hline 30 & 40133010 & -112.12 & 33.46 \\
\hline 31 & 40278011 & -114.61 & 32.69 \\
\hline 32 & 51190007 & -92.28 & 34.76 \\
\hline 33 & 60070008 & -121.84 & 39.76 \\
\hline 34 & 60090001 & -120.68 & 38.2 \\
\hline 35 & 60510005 & -119.12 & 37.96 \\
\hline 36 & 60970001 & -123.02 & 38.8 \\
\hline 37 & 60990005 & -120.99 & 37.64 \\
\hline 38 & 120570083 & -82.38 & 27.86 \\
\hline 39 & 150030010 & -158.09 & 21.32 \\
\hline 40 & 300290009 & -114.34 & 48.4 \\
\hline 41 & 300710010 & -107.86 & 48.32 \\
\hline 42 & 410390059 & -123.14 & 44.07 \\
\hline 43 & 420030003 & -79.77 & 40.45 \\
\hline 44 & 530330080 & -122.31 & 47.57 \\
\hline 45 & 530630021 & -117.36 & 47.67 \\
\hline 46 & 560350100 & -110.06 & 42.79 \\
\hline 47 & 560370007 & -109.22 & 41.59 \\
\hline 48 & 560370300 & -109.79 & 41.75 \\
\hline 49 & 560370866 & -109.79 & 41.63 \\
\hline 50 & 560370867 & -108.67 & 41.75 \\
\hline 51 & 10030010 & -87.88 & 30.5 \\
\hline 52 & 21220008 & -151.07 & 60.49 \\
\hline 53 & 40011235 & -109.44 & 35.88 \\
\hline 54 & 50010011 & -91.56 & 34.52 \\
\hline
\end{tabular}




\begin{tabular}{|c|c|c|c|}
\hline 55 & 60010007 & -121.78 & 37.69 \\
\hline 56 & 60410001 & -122.52 & 37.97 \\
\hline 57 & 60670006 & -121.37 & 38.61 \\
\hline 58 & 60710306 & -117.33 & 34.51 \\
\hline 59 & 60730003 & -116.94 & 32.79 \\
\hline 60 & 60731006 & -116.77 & 32.84 \\
\hline 61 & 60750005 & -122.4 & 37.77 \\
\hline 62 & 60771002 & -121.27 & 37.95 \\
\hline 63 & 61111004 & -119.23 & 34.45 \\
\hline 64 & 100010002 & -75.56 & 38.99 \\
\hline 65 & 131210039 & -84.44 & 33.8 \\
\hline 66 & 131350002 & -84.07 & 33.96 \\
\hline 67 & 150011006 & -155.11 & 19.72 \\
\hline 68 & 160410001 & -111.81 & 42.01 \\
\hline 69 & 330115001 & -71.88 & 42.86 \\
\hline 70 & 380070002 & -103.38 & 46.89 \\
\hline 71 & 471570047 & -90.02 & 35.17 \\
\hline 72 & 550090005 & -87.99 & 44.51 \\
\hline 73 & 560290001 & -109.07 & 44.53 \\
\hline 74 & 20900034 & -147.73 & 64.85 \\
\hline 75 & 40070009 & -110.86 & 33.4 \\
\hline 76 & 60010011 & -122.28 & 37.81 \\
\hline 77 & 80013001 & -104.95 & 39.84 \\
\hline 78 & 90010012 & -73.16 & 41.2 \\
\hline 79 & 120110010 & -80.17 & 26.13 \\
\hline 80 & 120170006 & -82.64 & 28.96 \\
\hline 81 & 160050004 & -112.52 & 42.92 \\
\hline 82 & 170191001 & -88.37 & 40.05 \\
\hline 83 & 220150008 & -93.75 & 32.54 \\
\hline 84 & 230090103 & -68.26 & 44.38 \\
\hline 85 & 230112005 & -69.79 & 44.23 \\
\hline 86 & 230310009 & -70.77 & 43.11 \\
\hline 87 & 240053001 & -76.47 & 39.31 \\
\hline 88 & 271095008 & -92.45 & 44 \\
\hline 89 & 300490004 & -111.99 & 46.85 \\
\hline 90 & 390350038 & -81.68 & 41.48 \\
\hline 91 & 390810017 & -80.62 & 40.37 \\
\hline 92 & 390850003 & -81.42 & 41.67 \\
\hline 93 & 401430235 & -96 & 36.13 \\
\hline \multicolumn{4}{|c|}{ CAWNET } \\
\hline 1 & Chengdu & 104.3 & 30.6 \\
\hline 2 & Dalian & 121.6 & 38.9 \\
\hline 3 & Dunhuang & 94.67 & 40.1 \\
\hline 4 & Gaolanshan & 105.8 & 36 \\
\hline
\end{tabular}




\begin{tabular}{|c|c|c|c|}
\hline & & & \\
\hline 5 & Gucheng & 115.8 & 39.1 \\
\hline 6 & Jinsha & 114.2 & 29.6 \\
\hline 7 & LinAn & 119.7 & 30.3 \\
\hline 8 & Longfengshan & 127.5 & 44.7 \\
\hline 9 & Shangdianzi & 117.1 & 40.7 \\
\hline 10 & Taiyangshan & 111.7 & 29.2 \\
\hline 11 & Xian & 109 & 34.5 \\
\hline 12 & Zhenbeitai & 109.2 & 38.5 \\
\hline 13 & Zhengzhou & 113.7 & 34.8 \\
\hline \multicolumn{4}{|c|}{ CNEMC } \\
\hline 1 & 110000041 & 116.17 & 40.29 \\
\hline 2 & 110000244 & 116.43 & 39.95 \\
\hline 3 & 110000245 & 116.43 & 39.87 \\
\hline 4 & 110000246 & 116.4 & 39.98 \\
\hline 5 & 110000247 & 116.47 & 39.97 \\
\hline 6 & 110000249 & 116.22 & 39.93 \\
\hline 7 & 110000250 & 116.36 & 39.94 \\
\hline 8 & 110000251 & 116.32 & 39.99 \\
\hline 9 & 110000252 & 116.37 & 39.87 \\
\hline 10 & 110000253 & 116.72 & 40.14 \\
\hline 11 & 110000254 & 116.64 & 40.39 \\
\hline 12 & 110000255 & 116.23 & 40.2 \\
\hline 13 & 440100051 & 113.24 & 23.14 \\
\hline 14 & 440100057 & 113.26 & 23.13 \\
\hline 15 & 440100063 & 113.28 & 23.16 \\
\hline 16 & 440100064 & 113.26 & 23.1 \\
\hline 17 & 440100073 & 113.32 & 23.14 \\
\hline 18 & 440100088 & 113.35 & 23.09 \\
\hline 19 & 440100089 & 113.43 & 23.1 \\
\hline 20 & 440100090 & 113.35 & 22.95 \\
\hline 21 & 440100091 & 113.21 & 23.39 \\
\hline 22 & 440100092 & 113.57 & 23.28 \\
\hline 23 & 442000051 & 113.38 & 22.52 \\
\hline 24 & 442000052 & 113.39 & 22.55 \\
\hline 25 & 442000053 & 113.41 & 22.51 \\
\hline 26 & 131000402 & 116.68 & 39.52 \\
\hline 27 & 131000403 & 116.77 & 39.57 \\
\hline 28 & 131000407 & 116.71 & 39.56 \\
\hline 29 & 131000408 & 116.75 & 39.53 \\
\hline 30 & 410100051 & 113.64 & 34.75 \\
\hline 31 & 410100052 & 113.6 & 34.75 \\
\hline 32 & 410100053 & 113.68 & 34.75 \\
\hline 33 & 410100054 & 113.64 & 34.77 \\
\hline 34 & 410100062 & 113.68 & 34.8 \\
\hline
\end{tabular}




\begin{tabular}{|c|c|c|c|}
\hline 35 & 410100063 & 113.56 & 34.8 \\
\hline 36 & 410100064 & 113.73 & 34.72 \\
\hline 37 & 410100065 & 113.73 & 34.72 \\
\hline 38 & 420100051 & 114.28 & 30.62 \\
\hline 39 & 420100052 & 114.15 & 30.48 \\
\hline 40 & 420100053 & 114.25 & 30.55 \\
\hline 41 & 420100054 & 114.3 & 30.55 \\
\hline 42 & 420100055 & 114.37 & 30.57 \\
\hline 43 & 420100056 & 114.43 & 30.61 \\
\hline 44 & 420100057 & 114.3 & 30.59 \\
\hline 45 & 420100075 & 114.39 & 30.48 \\
\hline 46 & 420100076 & 114.21 & 30.64 \\
\hline 47 & 320600073 & 120.86 & 32 \\
\hline 48 & 320600074 & 120.87 & 32.02 \\
\hline 49 & 320600077 & 120.94 & 31.93 \\
\hline 50 & 320600078 & 120.81 & 32.04 \\
\hline 51 & 320900401 & 120.12 & 33.4 \\
\hline 52 & 320900402 & 120.16 & 33.39 \\
\hline 53 & 320900403 & 120.13 & 33.37 \\
\hline 54 & 320900406 & 120.22 & 33.39 \\
\hline 55 & 310000051 & 121.4 & 31.24 \\
\hline 56 & 310000052 & 121.54 & 31.27 \\
\hline 57 & 310000053 & 121.48 & 31.2 \\
\hline 58 & 310000055 & 121.47 & 31.3 \\
\hline 59 & 310000056 & 121.43 & 31.23 \\
\hline 60 & 310000057 & 121.41 & 31.17 \\
\hline 61 & 310000058 & 121.53 & 31.23 \\
\hline 62 & 310000059 & 121.58 & 31.21 \\
\hline 63 & 310000251 & 121.7 & 31.19 \\
\hline 64 & 510100051 & 104.05 & 30.66 \\
\hline 65 & 510100052 & 104.03 & 30.65 \\
\hline 66 & 510100054 & 104.12 & 30.64 \\
\hline 67 & 510100064 & 104.07 & 30.68 \\
\hline 68 & 510100073 & 104.08 & 30.57 \\
\hline 69 & 510100074 & 104.18 & 30.69 \\
\hline 70 & 510100075 & 103.97 & 30.71 \\
\hline 71 & 120000051 & 117.15 & 39.1 \\
\hline 72 & 120000062 & 117.14 & 39.17 \\
\hline 73 & 120000072 & 117.18 & 39.12 \\
\hline 74 & 120000081 & 117.19 & 39.17 \\
\hline 75 & 120000095 & 117.24 & 39.11 \\
\hline 76 & 120000100 & 117.27 & 39.13 \\
\hline 77 & 120000104 & 117.2 & 39.09 \\
\hline 78 & 120000137 & 117.46 & 38.84 \\
\hline
\end{tabular}




\begin{tabular}{cccc}
\hline 79 & 120000143 & 117.71 & 39.03 \\
80 & 120000168 & 117.31 & 39.09 \\
81 & 120000186 & 117.18 & 39.23 \\
82 & 120000301 & 117.4 & 39.12 \\
83 & 120000302 & 117.76 & 39.16 \\
84 & 650100051 & 87.6 & 43.77 \\
85 & 650100055 & 87.58 & 43.83 \\
86 & 650100056 & 87.55 & 43.87 \\
87 & 650100071 & 87.64 & 43.83 \\
88 & 650100072 & 87.42 & 43.87 \\
89 & 650100091 & 87.64 & 43.96 \\
\hline \multicolumn{4}{c}{ Aerosol sites in China } \\
\hline 1 & Beijing & 116.371 & 39.974 \\
3 & Xinzhou & 112.12 & 38.07 \\
4 & Nanjing & 118.749 & 32.057 \\
\hline
\end{tabular}

Table S2. the budget of $\mathrm{O}_{3}$ and $\mathrm{CO}$ compared with the other models.

\begin{tabular}{|c|c|c|c|}
\hline Species & \multicolumn{2}{|c|}{ Process } & IAP-AACM \\
\hline \multirow{11}{*}{$\mathrm{CO}$} & \multirow{3}{*}{$\begin{array}{l}\text { Emission } \\
\left(\mathrm{Tg} \mathrm{yr}^{-1}\right)\end{array}$} & Anthrop. & 546.4 \\
\hline & & Bio. burning & 336.2 \\
\hline & & & \\
\hline & \multirow{2}{*}{ Total 994} & Biogenic & 92.7 \\
\hline & & Others & 18.3 \\
\hline & \multicolumn{2}{|c|}{ Top condition inflow $\left(\mathrm{Tg} \mathrm{yr}^{-1}\right)$} & 28 \\
\hline & \multicolumn{2}{|c|}{ Chem pro $\left(\mathrm{Tg} \mathrm{yr}^{-1}\right)$} & 1270 \\
\hline & \multicolumn{2}{|c|}{ Chem lss $\left(\operatorname{Tg~yr}^{-1}\right)$} & 2292 \\
\hline & \multicolumn{2}{|c|}{ Dry dep $\left(\mathrm{Tg} \mathrm{yr}^{-1}\right)$} & 0 \\
\hline & \multicolumn{2}{|l|}{ Burden $(\mathrm{Tg})$} & 327 \\
\hline & \multicolumn{2}{|l|}{ Lifetime (days) } & 52 \\
\hline \multirow{6}{*}{$\mathrm{O}_{3}$} & \multicolumn{2}{|c|}{ Top condition inflow $\left(\mathrm{Tg} \mathrm{yr}^{-1}\right)$} & 398 \\
\hline & \multicolumn{2}{|c|}{ Chemical production $\left(\mathrm{Tg} \mathrm{yr}^{-1}\right)$} & 4526 \\
\hline & \multicolumn{2}{|c|}{ Chemical loss $\left(\operatorname{Tg~yr}^{-1}\right)$} & 3875 \\
\hline & \multicolumn{2}{|c|}{ 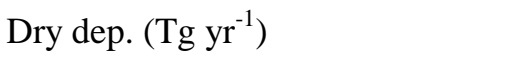 } & 1049 \\
\hline & \multicolumn{2}{|l|}{ Burden $(\mathrm{Tg})$} & 370 \\
\hline & \multicolumn{2}{|l|}{ Lifetime (days) } & 27.4 \\
\hline
\end{tabular}


Table S3. NMB of global domain in different regions. The NMB is calculated with annual average concentration. $\mathrm{ASO}_{4}, \mathrm{ANO}_{3}$ and $\mathrm{ANH}_{4}$ represents sulfate, nitrate and ammonium, respectively.

\begin{tabular}{lcccccccccc}
\hline & $\mathrm{CO}$ & $\mathrm{O}_{3}$ & $\mathrm{NO}_{2}$ & $\mathrm{SO}_{2}$ & $\mathrm{ASO}_{4}$ & $\mathrm{ANO}_{3}$ & $\mathrm{ANH}_{4}$ & $\mathrm{BC}$ & $\mathrm{OC}$ & $\mathrm{PM}_{2.5}$ \\
\hline Africa & -0.47 & -0.09 & & & & & & & & \\
Antarctica & -0.50 & -0.34 & & & & & & & & \\
ArcticO & -0.45 & & & & & & & & & \\
Asia & -0.34 & 0.94 & -0.14 & 0.05 & 0.36 & -0.61 & 0.85 & -0.4 & -0.67 & -0.36 \\
AtlanticO & -0.54 & 0.16 & & & & & & & & \\
Europe & -0.39 & 0.10 & 0.16 & 3.79 & 1.1 & 0.74 & 1.49 & -0.62 & -0.55 & -0.35 \\
IndianO & -0.53 & & & & & & & & & \\
NAmerica & -0.23 & -0.18 & -0.14 & 3.51 & 1.94 & 0.50 & -0.46 & 0.64 & -0.12 & 1.16 \\
Oceania & -0.45 & -0.04 & & & & & & & & \\
PacificO & -0.59 & 0.14 & & & & & & & & \\
SAmerica & -0.47 & -0.12 & & & & & & & & \\
\hline
\end{tabular}




\section{Figures}

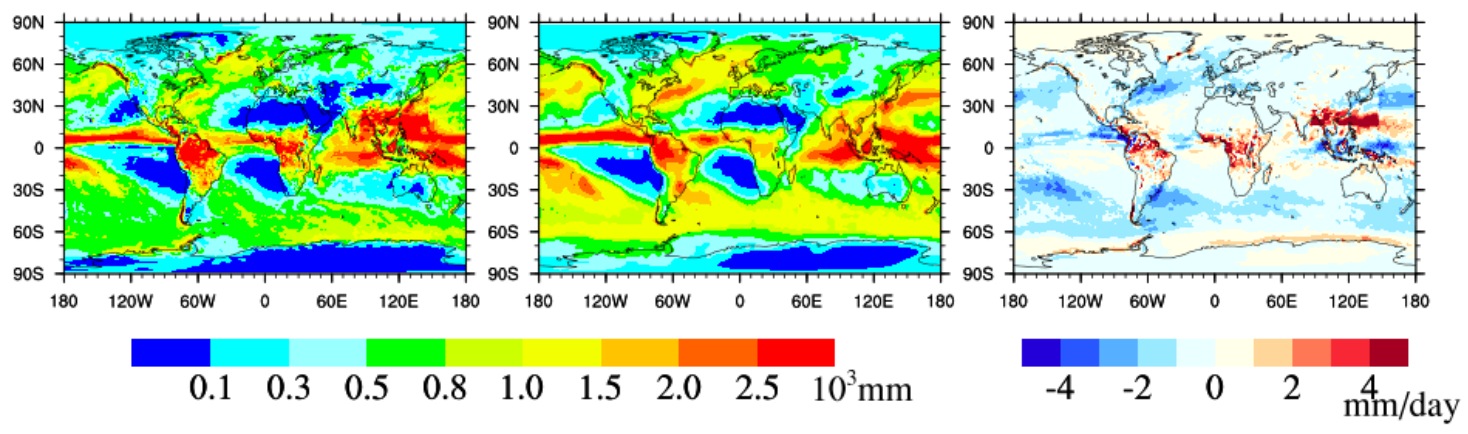

10 Fig. S1. Annual mean precipitation of WRF compared with GPCP data. The left column is WRF simulation (unit: $\mathrm{mm}$ ), the middle column is GPCP reanalysis data (unit: $\mathrm{mm}$ ), the right column is the difference between simulation and reanalysis (WRF-GPCP) (unit: $\mathrm{mm} \mathrm{day}^{-1}$ ).

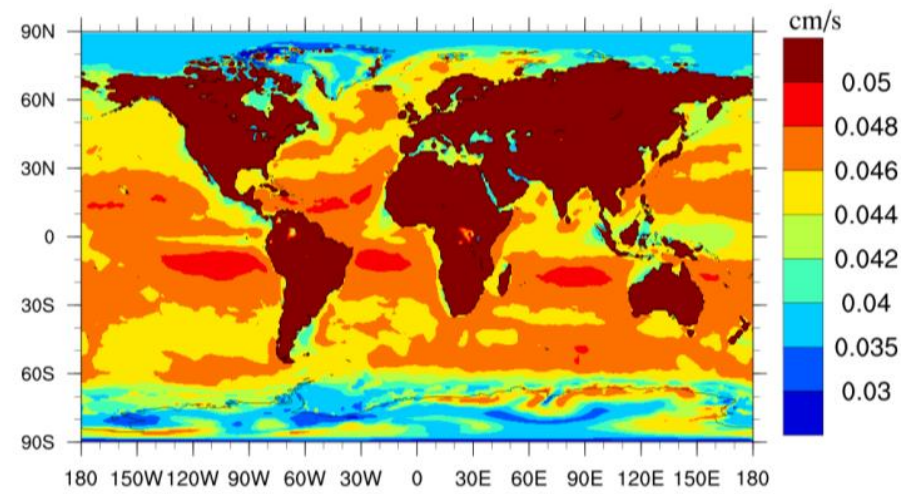

Fig. S2. Annual mean dry deposition velocity of ozone in IAP-AACM. The unit is $\mathrm{cm} \mathrm{s}^{-1}$.

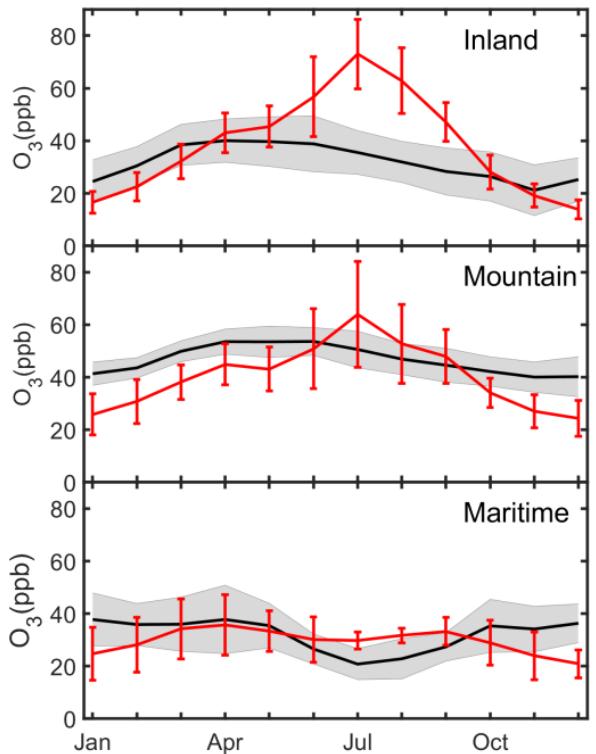

Fig. S3. Mean seasonal variation of $\mathrm{O}_{3}(\mathrm{ppb})$ over inland, mountain and maritime area 
in Northern Hemisphere compared with site records. Black lines and red lines represent the average of observations and simulations respectively. Gray shaded areas and red vertical bars show 1 standard deviation over the sites for observations and for model results respectively.

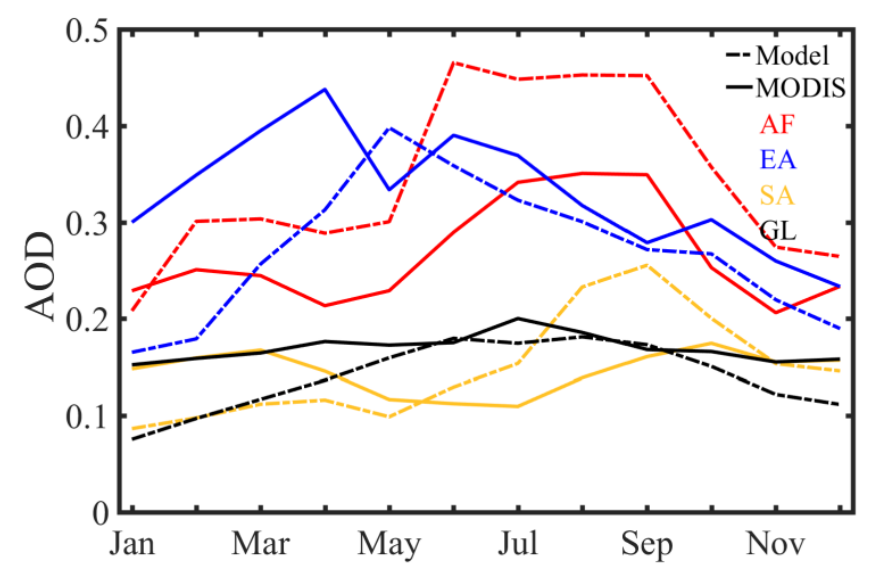

Fig. S4. Gridded mean value of monthly averaged AOD for 2014, AF, EA, SA and GL represents Africa, East Asia, South America and global. Dash line and solid line represents model results and observation derived from MODIS, respectively.

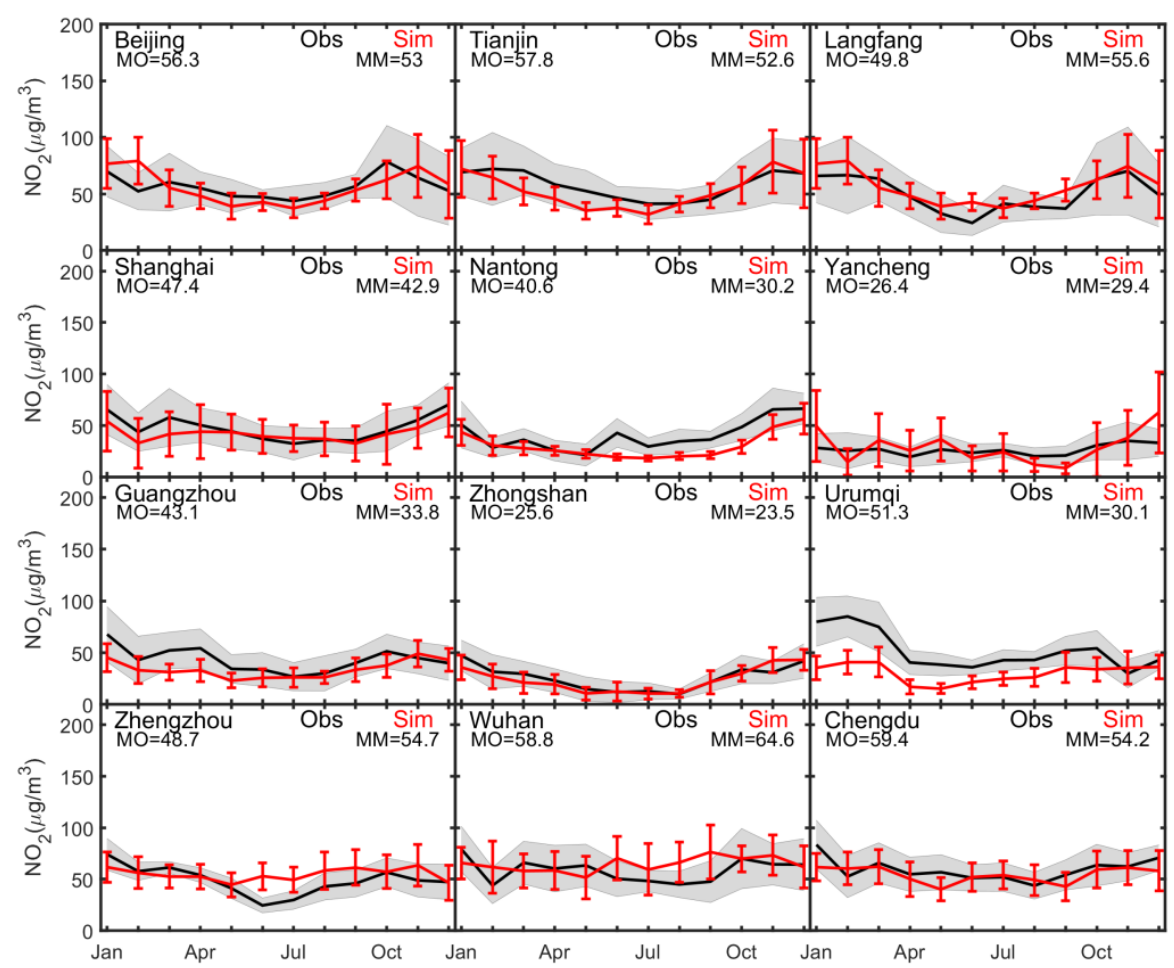

Fig. S5. Seasonal cycle of $\mathrm{NO}_{2}\left(\mu \mathrm{g} \mathrm{m}^{-3}\right)$ simulated without heterogeneous chemical process over China. The black line and red line represent monthly mean concentration of city-averaged observation and simulation respectively. Gray shaded areas and red 
vertical bars show 1 standard deviation over the sites for observations and for model results, respectively. MO and MM stand for annual mean concentration of observation and simulation respectively.
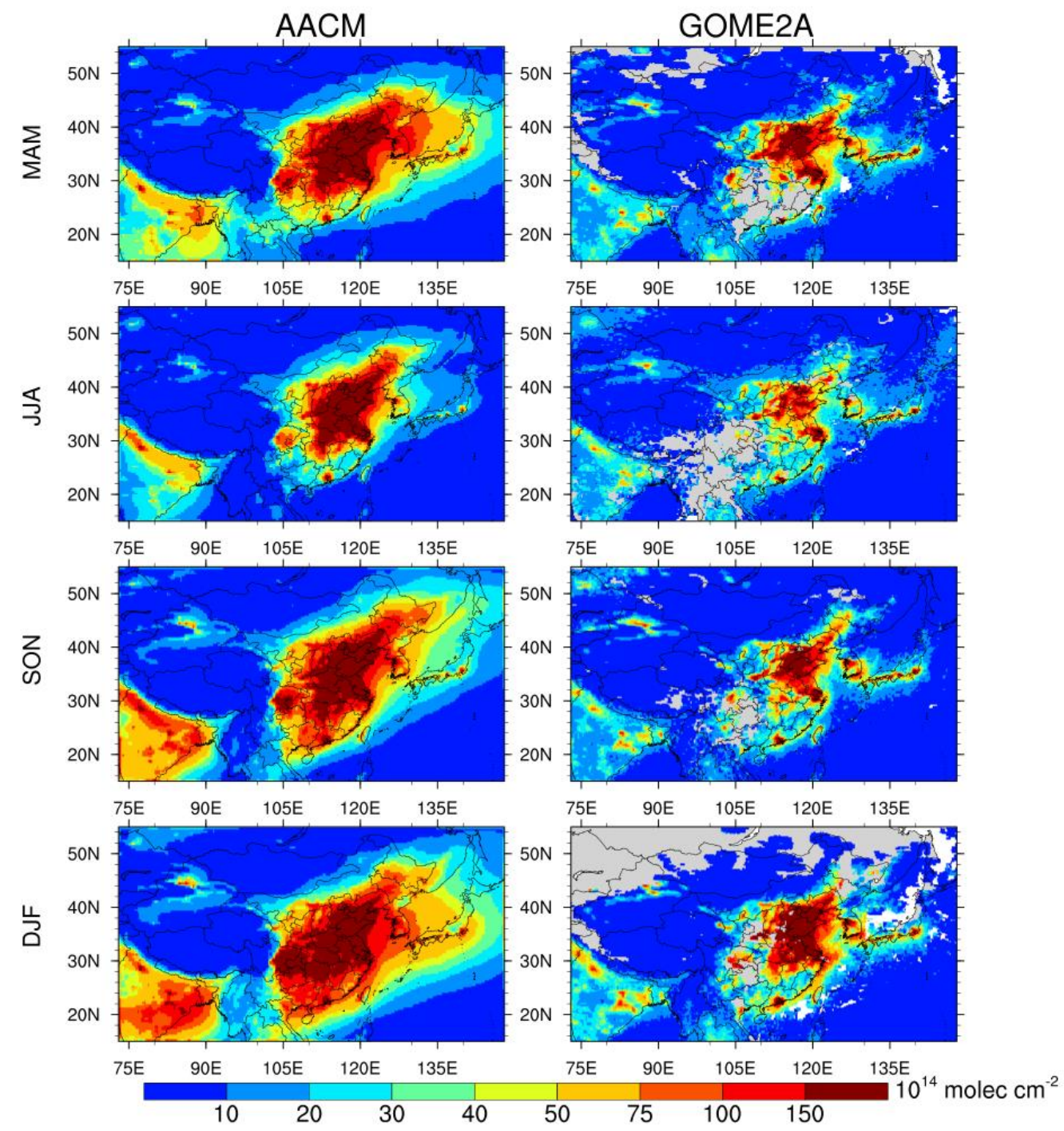

35 Fig. S6. Seasonal mean column concentration $\left(10^{14}\right.$ molecule $\left.\mathrm{cm}^{-2}\right)$ of $\mathrm{NO}_{2}$ in IAP-AACM and GOME-2A over China. 